\title{
ISO-ADIABATIC MERGING OF PBAR STACKS IN THE FERMILAB RECYCLER *
}

\author{
C. M. Bhat, \\ Fermi, Batavia, IL 60510, U.S.A. \\ (Presented at PAC2005)
}

\begin{abstract}
Considerable longitudinal emittance (LE) dilution is seen during merging of two stacks of pbars in the Fermilab Recycler. The emittance dilution results from the sequence of RF manipulations used for merging. Here I present a new scheme for merging two stacks adiabatically. This involves energy matching of the two stacks before merging. An analytical expression is derived for energy matching condition. The scheme is illustrated with multi-particle beam dynamics simulations and beam measurements. The beam experiments have shown that one can preserve the emittance to better than $15 \%$.
\end{abstract}

\section{INTRODUCTION}

The Recycler [1] is used as a second storage ring for anti-protons for collider shots at Fermilab. The antiprotons from the Accumulator are transferred periodically to the Recycler and cooled using stochastic cooling technique [2, 3]. A barrier RF system is used in the Recycler for injection and merging of stacks [4]. The maximum amount of pbar stack in the Recycler stored so far is about $200 \times 10^{10}$ pbars. We plan to store about $600 \times 10^{10}$ pbars once the Run II upgrades [5] including electron cooling (e-cool) $[6,7]$ of the pbars in the Recycler is complete. During the e-cool era, the newly arrived pbars will be cooled using stochastic cooling technique and the main stack will be cooled using e-cool technique. Subsequently, the new beam will be added to the main stack. It is highly essential to preserve the emittance of both of the stacks during the merging. The design criteria for e-cool [8] allow a maximum of $15 \%$ LE dilution during the merging process.

The scheme, we are presently using for merging two stacks [4] results in LE growth in excess of a factor of two. I have developed a new scheme which meets the design criteria $(<15 \%)$. The scheme has been successfully tested. I explain both of the schemes and ideas for future improvements.

\section{Current Scheme}

A schematic view of the current pbar stacking sequence is shown in Fig. 1. No effort is made to match the momentum spreads of the two stacks before merging. The "New Beam" is moved slowly towards the "Cold Beam" at a cog-rate of about $76 \mathrm{~ns} / \mathrm{s}$. As one of the positive RF pulse encounters a negative pulse (see

\footnotetext{
* Operated by Universities Research Association, Inc. for the U.S. Department of Energy under contract DE-AC02-76CH03000.

\#cbhat@fnal.gov
}

Fig.1b) of equal amplitude, they cancel one another resulting in zero amplitude. At that time an unstable region is created as shown in Fig. $1 \mathrm{~b}$ and 1c. The particles with largest energy deviation relative to the synchronous particles escape first and encounter unstable region. These beam particles tend to fly out of the bucket boundary contributing to the overall LE dilution. Besides, if we try to merge two stacks without momentum matching then the particles from the distribution with higher momentum spread penetrate the barrier earlier than the particles from second distribution and produce ring around the second distribution in $(\Delta E, \Delta t)$ phase space as shown in Fig. 1c. This leads to further emittance dilution.

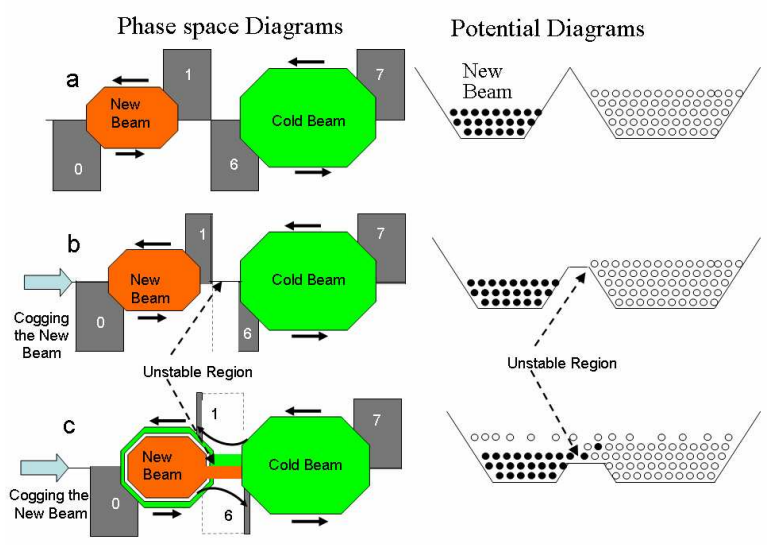

Figure 1. A schematic view of current bunch stacking sequence (with out energy matching before merging) in the Recycler and its potential diagram (right). The RF pulses are represented by regions $0,1,6$, and 7 .

\section{Proposed Scheme}

The first step in this scheme involves matching the energy spreads of the two stacks. In principle, this can be achieved by compressing or expanding adiabatically one of the beam distributions till its energy spread matches with that of the other. The second step is eliminating unstable region during merging. This is accomplished by bringing two stacks as close as possible and eliminating the RF voltage of in-between pulses adiabatically till the two stacks mix without emittance growth. A schematic view of various steps for this merging sequence is shown in Fig. 2. It is quite clear that the matching becomes technically more feasible if one fixes the locations of the RF pulses " 0 " and " 7 " while moving (cogging) intermediate pulses " 1 " and " 6 " together. This allows one to perform all RF 
manipulations within allotted area along the azimuth of a circular machine.

The method proposed here is one of several similar possibilities.

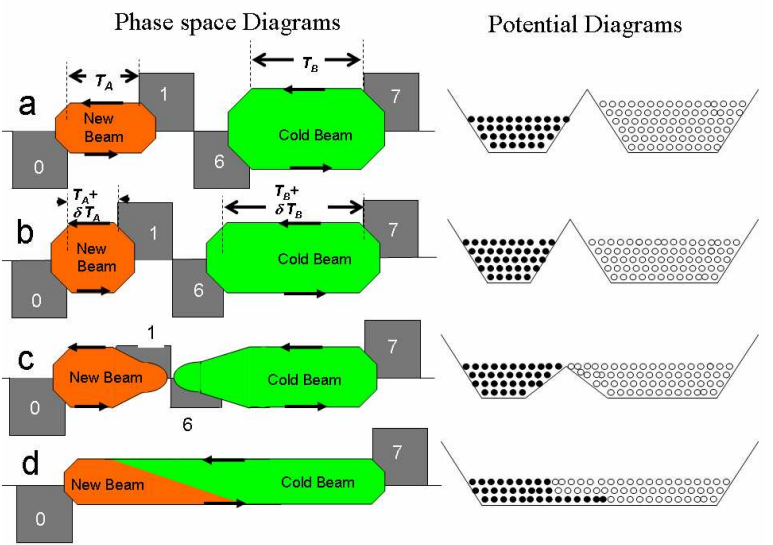

Figure 2. A schematic view of merging two stacks which would give no emittance growth. a) two stacks of beam with different energy spreads, b) after matching the energy spreads, c) intermediate stages of reducing heights of pulse 1 and 6 , d) final stage of merging. The corresponding potential diagrams are also shown on right side.

Here I derive an analytical expression for the required displacement, $\delta T_{A}$, for the intermediate pulses " 1 " and "6" to achieve matched energy spread for the case explained above. The LEs ( $\mathcal{E} \mathrm{s}$ ) of the two beam stacks are related to their pulse gaps $(T \mathrm{~s})$ and the measured (Schottky) energy spreads ( $\sigma$ s) according [9],

$$
\begin{aligned}
& \varepsilon_{A}=C_{1} T_{A} \sigma_{A}+C_{2} \sigma_{A}{ }^{3}+O \\
& \varepsilon_{B}=C_{1} T_{B} \sigma_{B}+C_{2} \sigma_{B}{ }^{3}+O
\end{aligned}
$$

The quantities $C_{1}$ and $C_{1}$ are constants which are functions of accelerator parameters. The adiabaticity condition calls for,

$$
\begin{aligned}
& \delta \varepsilon_{A}=0=\left(C_{1} T_{A}+3 C_{2} \sigma_{A}{ }^{2}\right) \delta \sigma_{A}+C_{1} \delta T_{A} \sigma_{A} \\
& \delta \varepsilon_{B}=0=\left(C_{1} T_{B}+3 C_{2} \sigma_{B}{ }^{2}\right) \delta \sigma_{B}+C_{1} \delta T_{B} \sigma_{B}
\end{aligned}
$$

for any change in $T_{A}$ and $T_{B}$. Solving for $\delta \sigma_{A}$ and $\delta \sigma_{B}$ we get,

$$
\begin{aligned}
& \delta \sigma_{A}=-\left[\frac{C_{1} \sigma_{A} \delta T_{A}}{C_{1} T_{A}+3 C_{2} \sigma_{A}{ }^{2}}\right] \\
& \delta \sigma_{B}=-\left[\frac{C_{1} \sigma_{B} \delta T_{B}}{C_{1} T_{B}+3 C_{2} \sigma_{B}{ }^{2}}\right]
\end{aligned}
$$

Now we impose that change in $T_{A}$ is same as $T_{B}$ and the energy spreads of two stacks after matching become equal. These two conditions give,

$$
\begin{aligned}
& \delta T_{A}=-\delta T_{B} \\
& \sigma_{A}^{\text {final }}=\sigma_{B} \text { final }
\end{aligned}
$$

The final energy spreads are given by,

$$
\begin{aligned}
& \sigma_{A}^{\text {final }}=\sigma_{A}+\delta \sigma_{A} \\
& \sigma_{B}^{\text {final }}=\sigma_{B}+\delta \sigma_{B}
\end{aligned}
$$

Then we can solve for $\delta T_{A}$ as,

$$
\begin{aligned}
& \delta T_{A} \\
& =\left[\frac{\left(\sigma_{B}-\sigma_{A}\right)\left(C_{1} T_{A}+3 C_{2} \sigma_{A}^{2}\right)\left(C_{1} T_{B}+3 C_{2} \sigma_{B}^{2}\right)}{C_{1} \sigma_{B}\left(C_{1} T_{A}+3 C_{2} \sigma_{A}^{2}\right)+C_{1} \sigma_{A}\left(C_{1} T_{B}+3 C_{2} \sigma_{B}^{2}\right)}\right]
\end{aligned}
$$

If $\delta T_{A}$ is positive then stack A needs to be compressed and if it is negative then stack A needs to be expanded to match the momentum spreads. The final merging is carried out by adiabatically lowering the heights of intermediate pulses.

It is important to note that the Eq. 6 has to be used with caution. For example if $T_{A}=T_{B}=0$ and $\sigma_{A}<$ $\sigma_{B}$ then the Eq. 6 gives non-zero values for $\delta T_{A}$, implying more compression, which is impractical. Under similar conditions one needd to match the energy spreads by expanding or compressing one of the stacks.

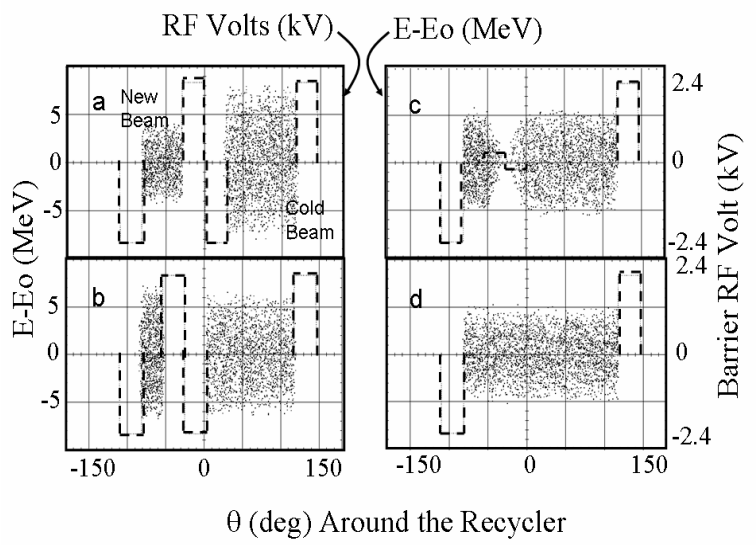

Figure 3. ESME simulations for proposed scheme a) two stacks of beam with different energy spreads, $\pm 4.4 \mathrm{MeV}$ (new beam) and $\pm 8.2 \mathrm{MeV}$ for (cold beam). In the present case the new and cold beams are just symbolic b) after matching the energy spreads, c) intermediate stages of reducing heights of pulse and d) final stage of merging.

\section{SIMULATIONS AND EXPERIMENTAL DEMONSTRATION}

I have carried out computer simulations of the schemes explained above using a multi-particle beam dynamics code, ESME [10]. The final demonstration is 
carried out with protons and anti-protons in the Recycler.

The simulations as well as experiments have been conducted with a barrier RF voltage $=2 \mathrm{kV}$, pulse width $=0.9 \mu$ s. The pulse gaps and LEs for stack A and B are taken to be $2.7 \mu \mathrm{s}$ and $1.6 \mu \mathrm{s}$ and, $48 \mathrm{eVs}$ and $14 \mathrm{eVs}$, respectively. Simulations on the current scheme show a minimum of about $30 \%$ longitudinal dilutions if the stacks are merged after energy matching. Without matching the dilution depends on energy spread difference and is much higher. During beam experiment we have observed about a factor of two LE dilutions.

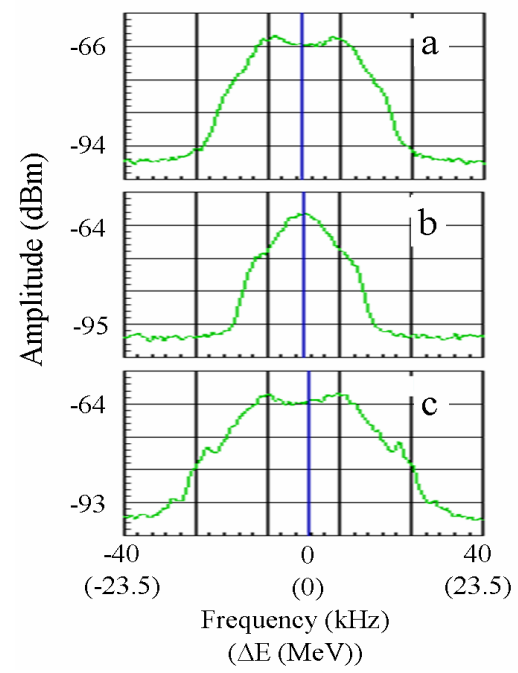

Figure 4. Schottky data for beam in barrier buckets of a) $T_{A}=2.7 \mu \mathrm{s}$, rms $\sigma_{A}=4.1 \mathrm{MeV}, \varepsilon_{A}=48 \mathrm{eVs} \mathrm{b)} T_{B}=1.59$ $\mu \mathrm{s}$, rms $\sigma_{B}=2.2 \mathrm{MeV} \quad \varepsilon_{B}=14 \mathrm{eVs}$, (before energy matching and merging,) and c) $T_{\text {final }}=3.27 \mu \mathrm{s}, \mathrm{rms} \sigma=4.8$ $\mathrm{MeV}, \varepsilon_{A}=68 \mathrm{eVs}$ (after merging using proposed scheme).

On the other hand, for the proposed scheme $\delta T_{A}=0.77 \mu$ s. Fig. 3 displays simulated $(\Delta \mathrm{E}, \theta)$-phase space distribution for this case. The final emittance after merging is found to be about $62 \mathrm{eVs}$ with $0 \%$ emittance growth.

We have conducted a series of experiments to test the proposed scheme for various initial beam intensities and longitudinal emittances. We found that one can keep the net emittance growth as low as $2 \%$. Fig. 4 shows Schottky data for a case which is a representative of the simulations results shown in Fig. 3. The initial LEs are $\varepsilon_{A}=(47 \pm 3)$ eVs (Fig. 4(a)) and $\varepsilon_{B}=(14 \pm 1)$ eVs (Fig. 4(b)) with Schottky measured rms energy spreads $(4.1 \pm 0.1)$ $\mathrm{MeV}$ and (2.2 \pm 0.1$) \mathrm{MeV}$, respectively. The energy spreads of these two distributions are matched by compressing distribution "B" by $0.77 \mu$ s (at the same time we expand the distribution " $A$ " by the same amount). Subsequently, these distributions are merged. The final emittance was $(68 \pm 3)$ eVs with rms energy spread (4.8 \pm 0.1$) \mathrm{MeV}$. Thus we find about $11 \%$ emittance dilution. The total time taken for the RF manipulation is found to be about $95 \mathrm{sec}$, with about 10 $\mathrm{sec}$ for matching the energy spreads, $25 \mathrm{sec}$ for bringing down the RF voltage and the rest for final compression. I believe that the small emittance dilution occurred during the final compression. Thus the measurements are consistent with the ESME simulation results presented earlier.

Another possible merging scheme is the reverse sequence of longitudinal momentum mining [11].

\section{REFERENCES}

[1] G. Jackson, Fermilab-TM-1991, November, 1996 (Unpublished).

[2] D. Mohl, et al, Phys. Rep. 58 (1980) 75; D. Mohl, Advanced Accelerator Physics Course, Proc. 1985 CERN Accelerator School, Ed. S. Turner, CERN 873 (1986) 453;

[3] D.R. Broemmelsiek et al, EPAC2004, page 794 ; "Gating System for Recycler $1.75 \mathrm{GHz}$ Schottky Detector," Ed. Cullerton and Ralph Pasquinelli, Fermilab Accelerator Division RF department Technical note- 062 (unpublished)

[4] C.M. Bhat, High Intensity and High Brightness Hadron Beam, edited by W. Chou et al, AIP Proceedings Vol. 642 (2002) 229; J. P. Marriner and the author jointly developed the current stack merging sequence for the Recycler (2002-2003).

[5] P.C. Bhat and W. J. Spalding, Hadron Collider Physics, HCP2004, edited by H. Weerts, AIP Proceedings Vol.753 (2004) p 30.

[6] G.I. Budker and A. N. Skrinsky, Sov. Phys.-Usp. 21 (1878) 277.

[7] J.R. Leibfritz et al, EPAC2004, page 1485.

[8] "Recycler Commissioning Plan", S. Nagaitsev and C. Gattuso, edited by P.C. Bhat, February 8, 2004, Beams-doc-1812-v1 (unpublished document).

[9] S.Y. Lee and K.Y. Ng, Phys. Rev. E 55, 5992 (1997); "Energy Spread and Emittance of a Beam in the Recycler," V. Balbekov, Beams-doc-1077-v1, Jan. 2004.

[10] J.A. MacLachlan, HEACC'98, Int. Conf. on High Energy Accelerators, Dubna, Sept. 1998; J. A. MacLachlan, Fermilab Report No FN-529, (1989).

[11]C.M. Bhat, Phys. Letts. A330 (2004) 481. 